\title{
System Optimization for Temporal Correlated Cognitive Radar with EBPSK-Based MCPC Signal
}

\author{
Peng Chen and Lenan Wu \\ School of Information Science and Engineering, Southeast University, Nanjing 210096, China \\ Correspondence should be addressed to Peng Chen; chenpengdsp@seu.edu.cn
}

Received 19 August 2014; Accepted 21 September 2014

Academic Editor: Yudong Zhang

Copyright ( 2015 P. Chen and L. Wu. This is an open access article distributed under the Creative Commons Attribution License, which permits unrestricted use, distribution, and reproduction in any medium, provided the original work is properly cited.

\begin{abstract}
The system optimization is considered in cognitive radar system (CRS) with extended binary phase shift keying- (EBPSK-) based multicarrier phase-coded (MCPC) signal. A novel radar working scheme is proposed to consider both target detection and estimation. At the detection stage, the generalized likelihood ratio test (GLRT) threshold is deduced, and the GLRT detection probability is given. At the estimation stage, an approach based on Kalman filtering (KF) is proposed to estimate target scattering coefficients (TSC), and the estimation performance is improved significantly by exploiting the TSC temporal correlation. Additionally, the optimal waveform is obtained to minimize the mean square error (MSE) of KF estimation. For the practical consideration, iteration algorithms are proposed to optimize the EBPSK-based MCPC signal in terms of power allocation and coding matrix. Simulation results demonstrate that the KF estimation approach can improve the estimation performance by $25 \%$ compared with maximum a posteriori MAP (MAP) method, and the KF estimation performance can be further improved by $90 \%$ by optimizing the transmitted waveform spectrum. Moreover, by optimizing the power allocation and coding matrix of the EBPSK-based MCPC signal, the KF estimation performances are, respectively, improved by $7 \%$ and $8 \%$.
\end{abstract}

\section{Introduction}

The cognitive radar system (CRS) as the future trend of the radar systems, compared with the traditional ones, mainly includes three different aspects [1]: (1) CRS can sense the targets and environment; (2) the transmitted waveform is adaptively optimized to improve the detection and estimation performance; (3) the transmitter, environment, and receiver form a closed loop feedback system. Therefore, optimizing the transmitted waveform based on the working environment becomes a popular research direction in the CRS [2-8].

For the modeling of CRS, a point is utilized to model the target [9]. The echo waveform from the point target is only the delay or Doppler frequency shift of the original one. However, when the size of target is large enough to occupy many resolution cells, the echo signals from different scattering cells are superimposed, and this type of target is modeled as an extended target (ET) [10]. In addition, the target impulse response (TIR), which is often referred to as the high resolution range profile (HRRP) in the automatic target recognition (ATR) problem [11], can be used to describe ET based on the assumption of the linear time-invariant target $[12,13]$. However, the view angle between the target and radar changes, and the ET does not satisfy this assumption. Hence, an exponential correlation model is proposed to describe this time dynamic characteristic, where the TIR is stationary with time and uncorrelated among different resolution cells, namely, wide sense stationary-uncorrelated scattering (WSSUS). Therefore, the TIR of this type target can be estimated by Kalman filtering (KF) [5, 14], where only the estimation is taken into consideration and under the present assumption of the target. However, the practical radar systems should detect the target before estimating.

The performance of the target estimation and detection can be significantly improved by optimizing the transmitted waveform in the CRS. During the stage to estimate the target scattering coefficients (TSC), which is essential the Fourier transform of TIR, the performance can be improved by optimizing the transmitted waveform to maximize the mutual information (MI) between the echo signal and TSC [12]. Furthermore, when the precise priori knowledge of 
the target cannot be obtained, an eigensubspace projectionbased method is proposed to enlarge the separation between the echo signals from different targets in $[15,16]$. During the detection stage, in order to maximize the probability of target detection, more power is concentrated on frequencies with relatively large TSC in the additive white Gaussian noise (AWGN) systems or relatively high ratio between the target and clutter in the clutter interference systems [17-20]. However, these optimized waveforms have arbitrary nature and are not conveniently generated in the practical radar systems.

The multicarrier phase-coded (MCPC) signal is first applied in the wideband radar systems by Levanon [21-23]. Furthermore, the MCPC waveform can achieve the optimal target detection performance with higher spectrum efficiency than the linear frequency modulation (LFM) signal [24, 25]. Unlike the P3 or P4 signal, the MCPC signal has a thumbtack-shaped ambiguity function and is easily generated in the practical radar systems. However, the nonconstant amplitude of MCPC waveform cannot take full advantage of the nonlinear amplifier [26]. On the modulation of MCPC waveform, an extended binary phase shift keying (EBPSK) modulation proposed by $\mathrm{Wu}$ et al. is adopted in this work $[27,28]$. EBPSK is more flexible than BPSK, where a small angle phase and jump time are utilized to distinguish the modulation waveform of code 1 from that of code 0 , which tightens the spectrum of the transmitted waveform $[25,29$, $30]$. In the wireless communication system, reference [31] has confirmed that EBPSK can achieve the same theoretical bit error rate (BER) performance as BPSK and achieve higher spectral efficiency with the same bit rate by tuning modulation parameters. Therefore, we first utilize the EBPSK-based MCPC signal in the CRS, since the tight spectrum is easy to optimize.

In this work, the problem of system optimization is considered in the CRS with EBPSK-based MCPC signal, and we propose a new radar working scheme, where both the target detection and estimation are taken into account. During the initial stage of the target detection, the generalized likelihood ratio test (GLRT) algorithm based on constant false alarm rate (CFAR) is utilized. In the presence of ET, the approach based on KF is proposed to estimate TSC by exploiting the temporal correlation, and the optimal spectrum is obtained to minimize the trace of the mean square error (MSE) matrix of KF estimation. In addition, the iteration algorithms are proposed to optimize the amplitudes and coding matrix of EBPSK-based MCPC signal, respectively.

The remainder of this work is organized as follows. In Section 2, the system model of the CRS with EBPSK-based MCPC signal and the new radar working scheme are given. In Section 3, we optimize this radar system, including the GLRT target detection based on CFAR, the maximum a posteriori probability (MAP) receive filter, the TSC estimation based on $\mathrm{KF}$, the optimal power allocation, and coding matrix. Section 4 gives the simulation results. Finally, Section 5 concludes this work.

The notations used in this work are defined as follows. Symbols for matrices (upper case) and vectors (lower case)

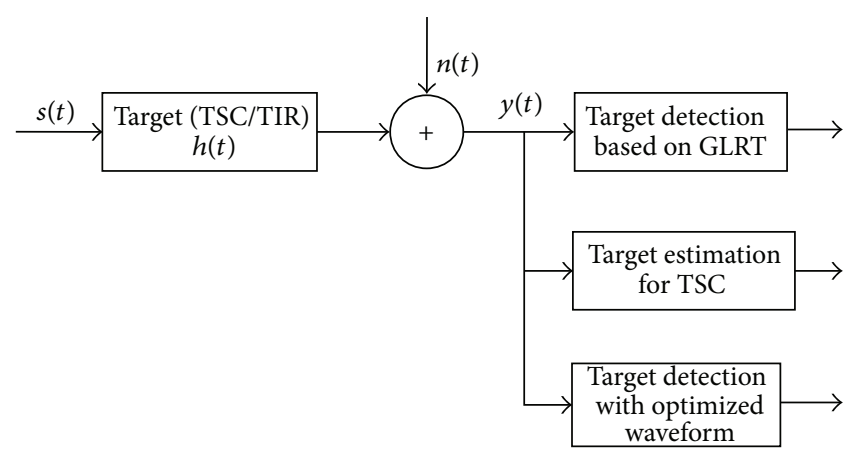

FIGURE 1: The system model of cognitive radar with EBPSK-based MCPC signal.

are in boldface. $(\cdot)^{H}, \operatorname{diag}\{\cdot\}, \mathbf{I}_{L}, \mathcal{N}(\mathbf{0}, \mathbf{R}),|\cdot|,\|\cdot\|_{2}, \operatorname{det}(\cdot)$, $\mathscr{E}\{\cdot\}$ and $\operatorname{Tr}\{\cdot\}$ denote the conjugate transpose (Hermitian), the diagonal matrix, the identity matrix of size $L$, the Gaussian distribution with zero mean and covariance being $\mathbf{R}$, the absolute value, the $\ell_{2}$ norm, the determinant of a matrix, the expectation, and the trace of a matrix, respectively.

\section{The System Model of Cognitive Radar with EBPSK-Based MCPC Signal}

The system model of cognitive radar considered in this work to detect and estimate the ET is shown in Figure 1, where the transmitted waveform is EBPSK-based MCPC signal $s_{k}(t)$ and the TIR of ET is $h_{k}(t)$ during the $k$ th pulse. Then the echo signal $y_{k}(t)$ can be described as

$$
y_{k}(t)=s_{k}(t) * h_{k}(t)+n_{k}(t), \quad k=1,2,3, \ldots,
$$

where $*$ denotes the convolution operation, and $n_{k}(t)$ denotes the AWGN. The EBPSK-based MCPC signal can be expressed as

$$
s_{k}(t)=\sum_{l=1}^{L} a_{k, l} \sum_{r=1}^{R} r_{k, l r}(t),
$$

where $r_{k, l r}(t) \triangleq C_{k, l r} s_{1}\left(t-T_{s} r, f_{l}\right)+\left(1-C_{k, l}\right) s_{0}(t-$ $\left.T_{s} r, f_{l}\right), C_{k, l r} \in\{0,1\}$ denotes the entry at $l$ th row and $r$ th column of the coding matrix $\mathbf{C}_{k}, a_{k, l}$ denotes the amplitude of the $l$ th subcarrier, and $f_{l} \triangleq f_{c}+(l-1) \Delta f$ denotes the carrier frequency of the $l$ th subcarrier, $f_{c}$ denotes the carrier frequency of the first subcarrier, and $\Delta f$ denotes the frequency interval between subcarriers. $s_{1}(t, f)$ and $s_{0}(t, f)$ are the waveform of code 0 and 1 with EBPSK modulation, respectively,

$$
\begin{aligned}
& s_{0}(t, f)=\cos (2 \pi f t), \quad 0 \leq t \leq T_{s} \\
& s_{1}(t, f)= \begin{cases}-\cos (2 \pi f t), & 0 \leq t \leq \tau \\
\cos (2 \pi f t), & \tau \leq t \leq T_{s},\end{cases}
\end{aligned}
$$

where $T_{s} \triangleq N / f_{c}(N=1,2,3, \ldots)$ denotes the time interval of the waveform for one code and $\tau \triangleq K / f_{c}(K=$ $1,2,3, \ldots, K \leq N$ ) denotes the jump interval of the EBPSK 
modulation. Then we can get the spectrum of transmitted waveform by the Fourier transform

$$
S_{k}(f)=\sum_{l=1}^{L} a_{k, l} \sum_{r=1}^{R} R_{k, l}(f) \exp \left(-j 2 \pi f T_{s} r\right),
$$

where $R_{k, l r}(f) \triangleq C_{k, l r} S_{1}\left(f, f_{l}\right)+\left(1-C_{k, l r}\right) S_{0}\left(f, f_{l}\right)$ and $S_{i}\left(f, f_{l}\right)(i \in\{0,1\})$ is the Fourier transform of $s_{i}\left(t, f_{l}\right)$; then the spectrum of echo signal $Y(f)$ is

$$
Y_{k}(f)=H_{k}(f) S_{k}(f)+N_{k}(f) .
$$

The discrete form in the frequency domain can be obtained as

$$
\mathbf{y}_{k}=\mathbf{S}_{k} \mathbf{g}_{k}+\mathbf{n}_{k},
$$

where the length of $\mathbf{y}_{k}$ is $M, \mathbf{n}_{k} \sim \mathcal{N}\left(\mathbf{0}, \sigma_{n}^{2} \mathbf{I}_{M}\right)$ denotes the discrete Gaussian noise, $\sigma_{n}^{2}$ denotes the variance of noise, $\mathbf{y}_{k}$ denotes the discrete form of $Y_{k}(f), \mathbf{g}_{k}$ denotes the TSC, that is, the discrete form of $H_{k}(f)$, and the diagonal matrix $\mathbf{S}_{k} \triangleq$ $\operatorname{diag}\left\{\mathbf{s}_{k}\right\}$, where $\mathbf{s}_{k}$ is the discrete form of $S_{k}(f)$. According to [14], the exponential correlation model of the TSC is

$$
\mathbf{g}_{k}=e^{-T / \tau} \mathbf{g}_{k-1}+\mathbf{u}_{k-1},
$$

where $\mathbf{g}_{k}$ denotes the TSC during the $k$ th pulse, $T$ denotes the pulse repetition interval (PRI) in the radar system, $\tau$ denotes the temporal decay constant, and $\mathbf{u}_{k-1} \sim \mathcal{N}\left(\mathbf{0},\left(1-e^{-T / \tau}\right) \boldsymbol{\Sigma}_{g}\right)$ follows the Gaussian distribution. When $\mathbf{g}_{0}=\mathbf{0}$,

$$
\mathbf{g}_{k}=\sum_{m=1}^{k-1} e^{-m T / \tau} \mathbf{u}_{k-1-m}
$$

and $\mathbf{g}_{k} \sim \mathcal{N}\left(\mathbf{0}, \mathbf{R}_{k} \triangleq\left(1-e^{-2 T / \tau}\right) \boldsymbol{\Sigma}_{g}\right)$. To simplify the analysis in this work, the correlation between the individual scatters is small enough to assume that $\Sigma_{g}$ is a diagonal matrix; then $\sigma_{k, G m}^{2}$ denotes the variance of $g_{k, m}$, where $g_{k, m}$ is the $m$ th entry of $\mathbf{g}_{k}$.

This work proposes a new radar working scheme for the CRS with EBPSK-based MCPC signal. As shown in Figure 2, the target detection and estimation stages in this radar system are described as follows.

(1) Initially, the nonoptimized EBPSK-based MCPC signal with power evenly distributed across all subcarriers is transmitted, and the coding matrix is a random \pm 1 matrix.

(2) The CRS detects the target via the method based on GLRT, and the detection threshold is obtained by the theory of CFAR. If the power of echo signal is greater than threshold, then the CRS detects the presence of target, otherwise not.

(3) When the GLRT detects the presence of target, we propose an approach to estimate the TSC from the echo signal based on KF.

(4) In order to improve the performance of KF estimation, this work optimizes the EBPSK-based MCPC signal in terms of power allocation among subcarriers and coding matrix;

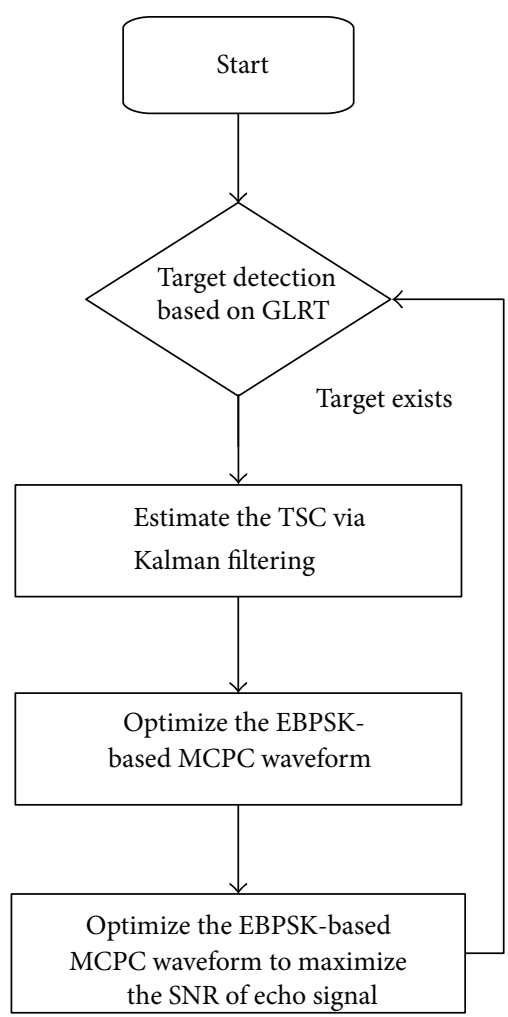

FIGURE 2: The proposed working scheme for the CRS with EBPSKbased MCPC signal.

(5) With the knowledge of the estimated TSC, the signalto-noise ratio (SNR) of the echo signal can be further improved, which attains a better detection probability for the particular target.

More details to describe this working scheme will be given in the following sections.

\section{The Optimization of CRS with EBPSK-Based MCPC Signal}

3.1. The Initial Target Detection Based on GLRT. Assuming that the target is present $H_{1}$ and absent $H_{0}$, the echo waveform is

$$
\begin{aligned}
& H_{0}: \mathbf{y}_{k}=\mathbf{n}_{k}, \\
& H_{1}: \mathbf{y}_{k}=\mathbf{S}_{k} \mathbf{g}_{k}+\mathbf{n}_{k} .
\end{aligned}
$$

With the Gaussian assuming of $\mathbf{g}_{k}$ and $\mathbf{n}_{k}$, the distribution of echo signal is also Gaussian, and the logarithmic likelihood ratio is

$$
\begin{aligned}
l\left(\mathbf{y}_{k} \mid \mathbf{g}_{k}\right) & \triangleq \ln \frac{p\left(\mathbf{y}_{k} \mid \mathbf{g}_{k}, H_{1}\right)}{p\left(\mathbf{y}_{k} \mid H_{0}\right)} \\
& =\frac{1}{\sigma_{n}^{2}}\left(2 \mathbf{y}_{k}^{H}-\mathbf{g}_{k}^{H} \mathbf{S}_{k}^{H}\right) \mathbf{S}_{k} \mathbf{g}_{k},
\end{aligned}
$$

where $p\left(\mathbf{y}_{k} \mid H_{0}\right)$ is the conditional probability distribution, and the constant term is eliminated in the second 
equation of (10). According to the GLRT theory, without the knowledge of TIR, the detection process can be expressed as

$$
\left\{l\left(\mathbf{y}_{k}\right) \triangleq l\left(\mathbf{y}_{k} \mid \overline{\mathbf{g}}_{k}\right)\right\} \underset{H_{0}}{\stackrel{H_{1}}{\gtrless}} \lambda,
$$

where

$$
\begin{aligned}
\overline{\mathbf{g}}_{k} & =\arg \max _{\mathbf{g}_{k}} l\left(\mathbf{y}_{k} \mid \mathbf{g}_{k}\right) \\
& =\arg \max _{\mathbf{g}_{k}}\left\|\mathbf{y}_{k}-\mathbf{S}_{k} \mathbf{g}_{k}\right\|_{2}=\mathbf{S}_{k}^{-1} \mathbf{y}_{k} .
\end{aligned}
$$

Then we can obtain the GLRT detection

$$
l\left(\mathbf{y}_{k}\right)=\frac{1}{\sigma_{n}^{2}} \mathbf{y}_{k}^{H} \mathbf{y}_{k} \underset{H_{0}}{\stackrel{H_{1}}{\gtrless}} \lambda .
$$

Therefore, without the knowledge of TSC, the GLRT detection is equivalent to the energy detecting. If the energy $\left\|\mathbf{y}_{k}\right\|_{2}^{2}$ is greater than the threshold $\lambda \sigma_{n}^{2}$, the target exists, otherwise not.

In the CRS, CFAR is adopted as the target detection criterion, and the echo signal meets the $\chi^{2}$ distribution with $M$ degrees of freedom when the target is absent, so the false alarm rate is

$$
\begin{aligned}
P_{F A} & =P\left(l\left(\mathbf{y}_{k}\right) \mid H_{0} \geq \lambda\right) \\
& =1-\frac{1}{\Gamma(M / 2)} \gamma\left(\frac{M}{2}, \frac{\lambda}{2}\right),
\end{aligned}
$$

where $\Gamma(x)$ is the gamma function and $\gamma(s, t)$ is the lower incomplete gamma function. When $\lambda>M$, the Chernoff bound of false alarm rate can be expressed as

$$
P_{F A} \leq\left(\frac{\lambda}{M} \exp \left(1-\frac{\lambda}{M}\right)\right)^{M / 2}
$$

Since the right side of (15) is the Lambert $W$ function, the solution is not easy to obtain, so we use the Taylor series to approximate it, and we can get the detection threshold

$$
\begin{aligned}
\lambda\left(P_{F A}\right) \approx & M P_{F A}^{2 / M}+M\left(1-P_{F A}^{2 / M}\right)-\frac{3 M}{2}\left(1-P_{F A}^{2 / M}\right) \\
& +\frac{8 M}{3}\left(1-P_{F A}^{2 / M}\right)^{4}-\frac{125 M}{24}\left(1-P_{F A}^{2 / M}\right)^{5} .
\end{aligned}
$$

When the target is present, the process of target detection is

$$
l\left(\mathbf{y}_{k}\right) \mid H_{1}=\frac{1}{\sigma_{n}^{2}}\left(\mathbf{S}_{k} \mathbf{g}_{k}+\mathbf{n}_{k}\right)^{H}\left(\mathbf{S}_{k} \mathbf{g}_{k}+\mathbf{n}_{k}\right) \underset{H_{0}}{\stackrel{H_{1}}{\gtrless}} \lambda\left(P_{F A}\right) .
$$

Since $s_{k, m} g_{k, m}+n_{k, m} \sim \mathcal{N}\left(0, s_{k, m}^{2} \sigma_{G m}^{2}+\sigma_{n}^{2}\right)$, where $s_{k, m}, g_{k, m}$, and $n_{k, m}$, respectively, denote the $m$ th entry of $\mathbf{s}_{k}, \mathbf{g}_{k}$, and $\mathbf{n}_{k}$, $x \triangleq l\left(\mathbf{y}_{k}\right) \mid H_{1}$ meets generalized chi-squared distribution and the probability density function is [32]

$$
f(x)=\sum_{m=1}^{M} \frac{\exp \left(-x / \xi_{m}^{2}\right)}{\xi_{m}^{2} \prod_{i=1, i \neq m}^{M}\left(1-\left(\xi_{i}^{2} / \xi_{m}^{2}\right)\right)},
$$

where $\xi_{m}^{2}=1+\left(s_{k, m}^{2} \sigma_{G m}^{2} / \sigma_{n}^{2}\right)$ and $\xi_{m}^{2} \neq \xi_{n}^{2}(\forall m \neq n)$. According to the Neyman_Pearson lemma, we can attain the probability of GLRT detection based on CFAR

$$
\begin{aligned}
P_{D} & =\int_{\lambda\left(P_{F A}\right)}^{\infty} f(x) d x \\
& =\sum_{m=1}^{M} \frac{\exp \left(-\lambda\left(P_{F A}\right) / \xi_{m}^{2}\right)}{\prod_{i=1, i \neq m}^{M}\left(1-\left(\xi_{i}^{2} / \xi_{m}^{2}\right)\right)} .
\end{aligned}
$$

3.2. The TSC Estimation Based on MAP. After detecting the presence of target, the TSC can be estimated from the echo waveform. When MAP is adopted as the receiver filter, we have

$$
\widehat{\mathbf{g}}_{k}=\arg \max _{\mathbf{g}_{k}} p\left(\mathbf{g}_{k} \mid \mathbf{y}_{k}\right)
$$

According to Bayes' theorem, we have

$$
p\left(\mathbf{g}_{k} \mid \mathbf{y}_{k}\right)=\frac{p\left(\mathbf{g}_{k}, \mathbf{y}_{k}\right)}{p\left(\mathbf{y}_{k}\right)}=\frac{p\left(\mathbf{y}_{k} \mid \mathbf{g}_{k}\right) p\left(\mathbf{g}_{k}\right)}{p\left(\mathbf{y}_{k}\right)},
$$

where $p\left(\mathbf{y}_{k} \mid \mathbf{g}_{k}\right)$ and $p\left(\mathbf{g}_{k}\right)$ are both the function of Gaussian distribution and the distribution of $\mathbf{y}_{k}$ is

$$
p\left(\mathbf{y}_{k}\right)=\int p\left(\mathbf{y}_{k} \mid \mathbf{g}_{k}\right) p\left(\mathbf{g}_{k}\right) d \mathbf{g}_{k} .
$$

We can obtain the posterior probability by substituting (22) into (21) as

$$
\begin{aligned}
p\left(\mathbf{g}_{k} \mid \mathbf{y}_{k}\right) & \\
= & \frac{p\left(\mathbf{y}_{k} \mid \mathbf{g}_{k}\right) p\left(\mathbf{g}_{k}\right)}{p\left(\mathbf{y}_{k}\right)} \\
= & \frac{\exp \left[\mathbf{g}_{k}^{H}\left(\left(1 / \sigma_{n}^{2}\right) \mathbf{S}_{k}^{H}\left(\mathbf{y}_{k}-(1 / 2) \mathbf{S}_{k} \mathbf{g}_{k}\right)-(1 / 2) \mathbf{R}_{k}^{-1} \mathbf{g}_{k}\right)\right]}{\exp \left(\left(1 / 2 \sigma_{n}^{4}\right) \mathbf{y}_{k}^{H} \mathbf{S}_{k} \mathbf{B}_{k}^{-H} \mathbf{S}_{k}^{H} \mathbf{y}_{k}\right) \sqrt{\operatorname{det}\left(\pi \mathbf{B}_{k}^{-1}\right)}},
\end{aligned}
$$

where $\mathbf{B}_{k} \triangleq\left(1 / \sigma_{n}^{2}\right) \mathbf{S}_{k}^{H} \mathbf{S}_{k}+\mathbf{R}_{k}^{-1}$ and $\mathbf{B}_{k}^{-H} \triangleq\left(\mathbf{B}_{k}^{H}\right)^{-1}$. We can get the optimization objective function by substituting (23) into (20) as

$$
\widehat{\mathbf{g}}_{k}=\arg \max _{\mathbf{g}_{k}}\left\{-\frac{1}{2} \mathbf{g}_{k}^{H} \mathbf{B}_{k} \mathbf{g}_{k}+\frac{1}{\sigma_{n}^{2}}\left(\mathbf{S}_{k} \mathbf{g}_{k}\right)^{H} \mathbf{y}_{k}\right\} .
$$

Therefore, the estimated TSC by MAP estimation is

$$
\widehat{\mathbf{g}}_{k}=\frac{1}{\sigma_{n}^{2}} \mathbf{B}_{k}^{-1} \mathbf{S}_{k}^{H} \mathbf{y}_{k} \text {. }
$$

The receiver filter can be written in the matrix form

$$
\mathbf{Q}_{k} \triangleq \frac{1}{\sigma_{n}^{2}} \mathbf{B}_{k}^{-1} \mathbf{S}_{k}^{H}
$$

Then the MSE matrix of the estimated TSC is

$$
\begin{aligned}
\mathbf{P}= & \mathscr{E}\left\{\left(\widehat{\mathbf{g}}_{k}-\mathbf{g}_{k}\right)\left(\widehat{\mathbf{g}}_{k}-\mathbf{g}_{k}\right)^{H}\right\} \\
= & \mathbf{Q}_{k}\left(\mathbf{S}_{k} \mathbf{R}_{k} \mathbf{S}_{k}^{H}+\sigma_{n}^{2} \mathbf{I}_{M}\right) \mathbf{Q}_{k}^{H} \\
& -\frac{1}{\sigma_{n}^{2}} \mathbf{B}_{k}^{-1} \mathbf{S}_{k}^{H} \mathbf{S}_{k} \mathbf{R}_{k}-\frac{1}{\sigma_{n}^{2}} \mathbf{R}_{k} \mathbf{S}_{k}^{H} \mathbf{S}_{k} \mathbf{B}_{k}^{-H}+\mathbf{R}_{k} .
\end{aligned}
$$


(1) Set the pulse index $k=1$, and the initial TSC estimation is based on MAP, where the estimated TSC $\widehat{\mathbf{g}}_{k \mid k}$ can be calculated from (25) and the MSE matrix $\mathbf{P}_{k \mid k}$ can be obtained from (27). Set the maximum number of pulses $K_{\max }$.

(2) Let $k=k+1$;

(3) while $k \leq K_{\max }$ and $k>1$ do

(4) According to the temporal correlation of TSC (4), the prediction of TSC is $\widehat{\mathbf{g}}_{k \mid k-1}=e^{-T / \tau} \widehat{\mathbf{g}}_{k-1 \mid k-1}$,

where $\widehat{\mathbf{g}}_{k-1 \mid k-1}$ is the estimated TSC during the $(k-1)$ th pulse;

(5) The MSE matrix of the predicted TSC is

$$
\mathbf{P}_{k \mid k-1}=e^{-2 T / \tau} \mathbf{P}_{k-1 \mid k-1}+\left(1-e^{-2 T / \tau}\right) \boldsymbol{\Sigma}_{g} ;
$$

(6) The KF gain matrix is defined as

$$
\boldsymbol{\Phi}_{k} \triangleq \mathbf{P}_{k \mid k-1} \mathbf{S}_{k}^{H}\left(\sigma_{n}^{2} \mathbf{Q}_{k}+\mathbf{Q}_{k} \mathbf{S}_{k} \mathbf{P}_{k \mid k-1} \mathbf{S}_{k}^{H}\right)^{-1} ;
$$

(7) Update the estimated TSC

$$
\widehat{\mathbf{g}}_{k \mid k}=\widehat{\mathbf{g}}_{k \mid k-1}+\boldsymbol{\Phi}_{k}\left(\widehat{\mathbf{g}}_{k}-\mathbf{Q}_{k} \mathbf{S}_{k} \widehat{\mathbf{g}}_{k \mid k-1}\right),
$$

where $\widehat{\mathbf{g}}_{k}=\mathbf{Q}_{k} \mathbf{y}_{k}$;

(8) Update the MSE matrix

(9) Let $k=k+1$.

$$
\mathbf{P}_{k \mid k}=\mathbf{P}_{k \mid k-1}-\boldsymbol{\Phi}_{k} \mathbf{Q}_{k} \mathbf{S}_{k} \mathbf{P}_{k \mid k-1}
$$

(10) end while

Algorithm 1: The TSC estimation based on KF.

3.3. The TSC Estimation Based on KF. We proposed an approach based on KF to estimate TSC according to the temporal correlation of TSC, when the GLRT detects the presence of target. Furthermore, we optimize the transmitted waveform to further improve the estimation performance by minimizing the trace of MSE matrix. The estimation approach based on KF is described in Algorithm 1.

In order to improve the performance of KF estimation, the trace of the MSE matrix $\mathbf{P}_{k \mid k}$ is utilized to measure this performance,

$$
f\left(\mathbf{s}_{k}\right)=\operatorname{Tr}\left\{\mathbf{P}_{k \mid k}\right\} .
$$

The transmitted waveform should be optimized to minimize the MSE at each iteration of KF, which is equivalent to the following optimization problem:

$$
\begin{array}{lc}
\mathbf{s}_{k}^{*}= & \arg \min _{\mathbf{s}_{k}} f\left(\mathbf{s}_{k}\right) \\
\text { s.t. } & \operatorname{Tr}\left\{\mathbf{S}_{k}^{H} \mathbf{S}_{k}\right\}=E_{s} \\
& \mathbf{S}_{k}=\operatorname{diag}\left\{\mathbf{s}_{k}\right\},
\end{array}
$$

where $E_{s}$ is the power of radar signal. The objective function can be simplified as

$$
\begin{aligned}
f\left(\mathbf{s}_{k}\right) & =\operatorname{Tr}\left\{\mathbf{P}_{k \mid k}\right\} \\
& =\sum_{m=1}^{M} \frac{\left|s_{m}\right|^{2} P_{k \mid k-1, m}^{2}}{\sigma_{n}^{2}+P_{k \mid k-1, m}\left|s_{m}\right|^{2}},
\end{aligned}
$$

where $P_{k \mid k-1, m}$ denotes the entry at $m$ th row and $m$ th column of $\mathbf{P}_{k \mid k}$. Then we can get the Lagrangian function of this optimization problem

$$
g\left(\mathbf{s}_{k}, \zeta\right)=f\left(\mathbf{s}_{k}\right)-\zeta\left(\sum_{m=1}^{M}\left|s_{m}\right|^{2}-E_{s}\right) .
$$

Let the derivation of (31) equal 0;

$$
\frac{\partial g\left(s_{k}, \zeta\right)}{\partial\left|s_{m}\right|^{2}}=0
$$

Therefore, the optimal spectrum of transmitted waveform should satisfy the following condition:

$$
\left|s_{m}^{*}\right|^{2}=\max \left\{0, \alpha \sqrt{\sigma_{n}^{2}}-\frac{\sigma_{n}^{2}}{P_{k \mid k-1, m}}\right\},
$$

where $\alpha$ is the constraint of transmitted power $\operatorname{Tr}\left(\mathbf{S}_{k}^{H} \mathbf{S}_{k}\right)=E_{s}$ and the optimal signal obtained in (34) is similar to the waterfilling method in the communication systems [12].

3.4. The Optimization of EBPSK-Based MCPC Signal. In the practical radar systems, the waveforms are generated by setting the modulation parameters or choosing from the preset waveforms, so it is difficult to attain the optimal waveform calculated in Section 3.3. Therefore, we propose a suboptimal signal by tuning the parameters of EBPSKbased MCPC waveform in terms of power allocation among subcarriers and coding matrix. In addition, the process of waveform optimization is simple while maintaining the estimation performance of TSC.

The optimal waveform obtained in (33) is referred as $\mathbf{s}_{k}^{*}$; then the best power allocation scheme for EBPSKbased MCPC signal should approximate the spectrum to this optimal one. Therefore, the MSE approximation can be expressed as an optimization problem

$$
\mathbf{s}_{k}^{\prime *}=\arg \max _{\mathbf{s}_{k}^{\prime}}=\left\|\left|\mathbf{s}_{k}^{\prime}\right|^{2}-\left|\mathbf{s}_{k}^{*}\right|^{2}\right\|_{2}^{2},
$$

where $\mathbf{s}_{k}^{\prime}$ denotes EBPSK-based MCPC signal in the frequency domain. Because of the sparsity of the EBPSK 
spectrum [30], we only need to consider the power allocation in carrier frequencies of subcarriers. The amplitudes should satisfy the following condition:

$$
\frac{a_{m}^{*}}{a_{n}^{*}}=\frac{\left|s_{k, m}^{*}\right|}{\left|s_{k, n}^{*}\right|}
$$

where $s_{k, m}^{*}$ donates the $m$ th entry of $\mathbf{s}_{k}^{*}$. The optimized power allocation (35) of EBPSK-based MCPC signal can improve the estimation performance of TSC via KF, and the simulation results will be given in Section 5 .

We will also optimize the coding matrix in the following, which can further reduce the MSE of the estimated TSC based on KF. The optimal coding matrix should be the solution of the following optimization problem:

$$
\begin{aligned}
& \mathbf{C}_{k}^{*}=\arg \min _{\mathbf{C}_{k}} \frac{\left|\widetilde{\mathbf{s}}_{k}^{H} \mathbf{x}\right|}{\left\|\widetilde{\mathbf{s}}_{k}\right\|_{2}\|\mathbf{x}\|_{2}} \\
& \text { s.t. } \quad C_{l r} \in\{0,1\},
\end{aligned}
$$

where $\widetilde{\mathbf{s}}_{k}$ denotes the discrete form the transmitted waveform $\widetilde{S}_{k}(f)=\sum_{l=1}^{L} a_{k, l} \sum_{r=1}^{R} C_{k, l} S_{1}\left(f, f_{l}\right)+(1-$ $\left.C_{k, l}\right) S_{0}\left(f, f_{l}\right) \exp \left(-j 2 \pi f T_{s} r\right)$ and $\mathbf{x}$ denotes the optimal reference signal, which will be obtained by the estimated TSC and the target scattering model in the following.

When the estimation value of scattering coefficients is $\widehat{\mathbf{g}}_{k-1 \mid k-1}$, the predicted value is

$$
\widehat{\mathbf{g}}_{k \mid k-1}=e^{-T / \tau} \widehat{\mathbf{g}}_{k-1 \mid k-1} .
$$

However, the real one is

$$
\mathbf{g}_{k}=e^{-T / \tau} \mathbf{g}_{k-1}+\mathbf{u}_{k-1} .
$$

We should design the transmitted signal $\mathbf{x}$, which minimizes the estimation MSE with the assumption of $\widehat{\mathbf{g}}_{k-1} \approx \mathbf{g}_{k-1}$. Since we have proposed the optimal power allocation scheme by the water-filling method, the estimation scattering vector is chosen as the optimal reference waveform, and (37) is the optimization objective function. Let

$$
\mathbf{x}=\widehat{\mathbf{g}}_{k \mid k-1}+(1+i) \sqrt{\frac{1}{2}\left(1-e^{-2 T / \tau}\right) \boldsymbol{\Sigma}_{g}}
$$

where $i$ is the imaginary unit. A simple iteration algorithm is proposed to calculate the optimal coding matrix, which is described in Algorithm 2.

\section{Simulation Results}

4.1. The TSC Estimation Based on MAP. First, we simulate the MSE of estimated TSC based on the MAP receiver filter and compare the simulation results with the theoretical ones. The parameters are set in Table 1, where the transmitted waveform is the EBPSK-based MCPC signal. In this work, all the simulation parameter are given based on the published literatures. For example, the parameters of EBPSK modulation are according to [20,21, 27-29], and the parameters for
TABLE 1: Simulation parameters.

\begin{tabular}{lc}
\hline Parameter & Value \\
\hline Carrier frequency $f_{c}$ & $100 \mathrm{MHz}$ \\
Sample frequency $f_{s}$ & $1 \mathrm{GHz}$ \\
The parameter of EBPSK modulation $K$ & 2 \\
The parameter of EBPSK modulation $N$ & 20 \\
The number of subcarriers $L$ & 16 \\
The length of codes of each subcarrier $N_{C}$ & 32 \\
Frequency interval $\Delta f$ & $20 \mathrm{MHz}$ \\
The SNR of echo signal & {$[-20,20] \mathrm{dB}$} \\
\hline
\end{tabular}

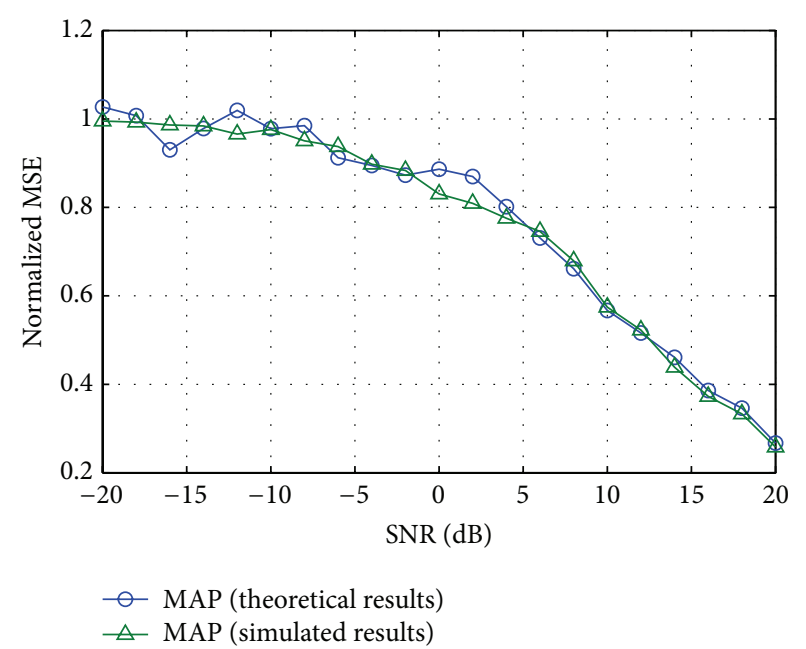

Figure 3: The estimation performance based on MAP.

the characteristics of target and radar are based on $[5,14]$. Although the realistic data is not adopted in our work, the simulation results are reliable and practicable.

Figure 3 depicts a comparison between theoretical and simulation results of the normalized MSE of estimated TSC, where the theoretical results are calculated by the trace of MSE matrix in (28), and the normalized MSE is defined as

$$
e\left(\widehat{\mathbf{g}}_{k}\right) \triangleq \frac{\left\|\widehat{\mathbf{g}}_{k}-\mathbf{g}_{k}\right\|_{2}^{2}}{\left\|\mathbf{g}_{k}\right\|_{2}^{2}} .
$$

We can observe from this figure that the simulation and theoretical results have a relatively high degree of agreement, which verifies the efficiency to measure the estimation performance of MAP by the trace of MSE matrix.

4.2. The TSC Estimation Based on KF. This subsection gives the simulation results of the estimated TSC based on $\mathrm{KF}$, in which the simulation parameters are the same with Section 4.1 except $\mathrm{SNR}=5 \mathrm{~dB}$, and the index of iteration is from 1 to 50. Figure 4 demonstrates the normalized MSE of estimated TSC, including the approach based on KF and MAP. Apparently, the estimation performance is improved about $25 \%$ by using the KF approach instead of MAP, since the temporal correlation is exploited by KF. In addition, the theoretical results given in (28) have high degree of 
(1) Initialize the coding matrix $\mathbf{C}^{*}$ with a random \pm 1 matrix, and set the iteration index $i=1$ and the maximum number of iterations $I_{\max }$;

(2) while $i \leq I_{\max }$ and the coding matrix changes do

(3) Let $r=i \bmod M L, C^{*}(\lfloor r / M\rfloor+1, r-M\lfloor r / M\rfloor)=1-C^{*}(\lfloor r / M\rfloor+1, r-M\lfloor r / M\rfloor)$, where $C^{*}(m, n)$ denotes the entry at the $m$ th row and $n$th column of $\mathbf{C}^{*}$;

(4) Generate the EBPSK-based MCPC waveform $\widetilde{\mathbf{s}}_{i}$ with coding matrix $\mathbf{C}^{*}$ and calculate

$$
z_{i}=\left\|\left|\widetilde{\mathbf{s}}_{i}\right|^{2}-|\mathbf{x}|^{2}\right\|_{2}^{2}
$$

(5) If $z_{i}>z_{i-1}, i=i+1$, and go to Step 3, otherwise, let $C^{*}(\lfloor r / M\rfloor+1, r-M\lfloor r / M\rfloor)=1-C^{*}(\lfloor r / M\rfloor+1, r-M\lfloor r / M\rfloor), i=i+1$ and go to Step 3;

(6) Let $i=i+1$.

(7) end while

Algorithm 2: The algorithm to optimize the coding matrix.

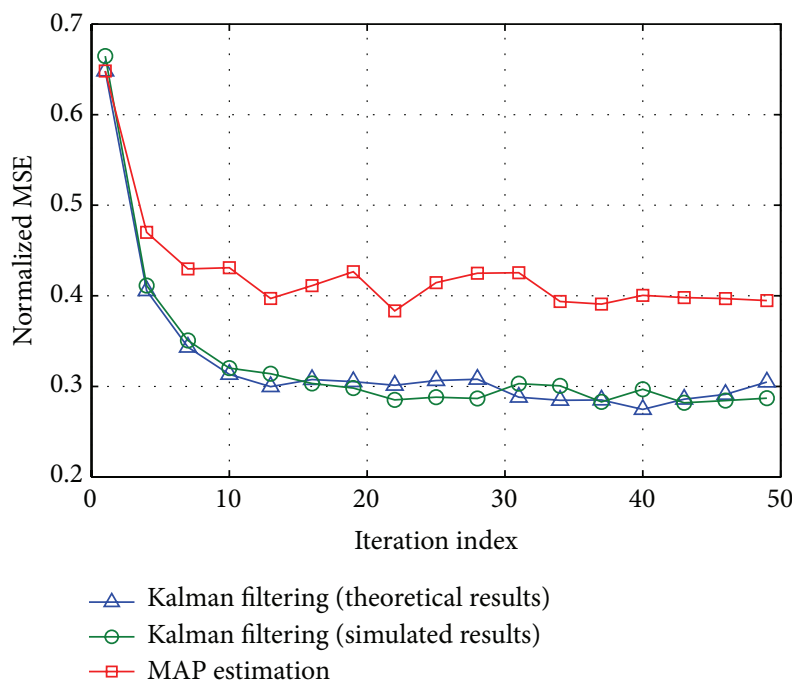

FIGURE 4: The estimation of TSC based on KF.

agreement with the simulation results. Therefore, the trace of MSE matrix can be utilized as an objective function, which accurately describes the estimation performance of $\mathrm{KF}$, in the waveform optimization problem. However, during the simulation, the decreasing normalized MSE of MAP estimation is caused by the exponential correlation model $\mathbf{g}_{k}=e^{-T / \tau} \mathbf{g}_{k-1}+\mathbf{u}_{k-1}$.

Figure 5 illustrates the spectrum of transmitted waveform, including the power uniform distributed EBPSKbased MCPC signal and the optimal spectrum. The optimal spectrum, which can minimize the trace of MSE matrix and improve the estimation performance of KF approach, is obtained by the water-filling method, where the parameter $\alpha$ is determined by the dichotomy algorithm. In addition, as shown in this figure, the variance of TSC is also given, which is similar to the optimal spectrum. Therefore, more power should be concentrated on the frequencies with relatively large variance of TSC, which provides guidance on the optimization of EBPSK-based MCPC signal.

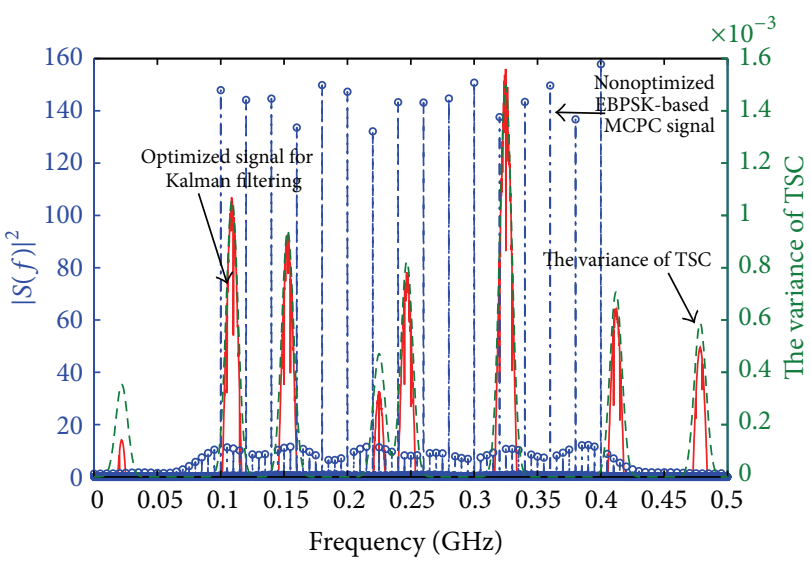

FIGURE 5: The optimized spectrum for the KF estimation.

Figure 6 shows the estimation performance of the methods based on MAP and KF, where the waveform with optimal spectrum and the random one is compared. We can observe that the simulated MSE and theoretical one have a relatively high degree of agreement. Moreover, the accuracy of estimation based on $\mathrm{KF}$ is more precise than the one based on MAP. In addition, optimizing the spectrum of transmitted waveform can further improve the estimation performance both by the approaches based on MAP and KF though the criterion of waveform optimization is the MSE matrix of the KF estimation. The performance of MAP estimation is improved by $90 \%$, and the performance of KF estimation is also improved by $90 \%$. Moreover, the KF estimation with optimized waveform is $18 \%$ better than the MAP one with optimized waveform.

4.3. The Optimization of EBPSK-Based MCPC Signal. Figure 7 depicts the spectrum of the optimal power allocation for EBPSK-based MCPC signal, where the KF estimation is adopted. The optimized power allocation shows that more power should be concentrated on the frequencies with relatively large variance of TSC. Therefore, we should design the EBPSK-based MCPC signal to approximate the signal 


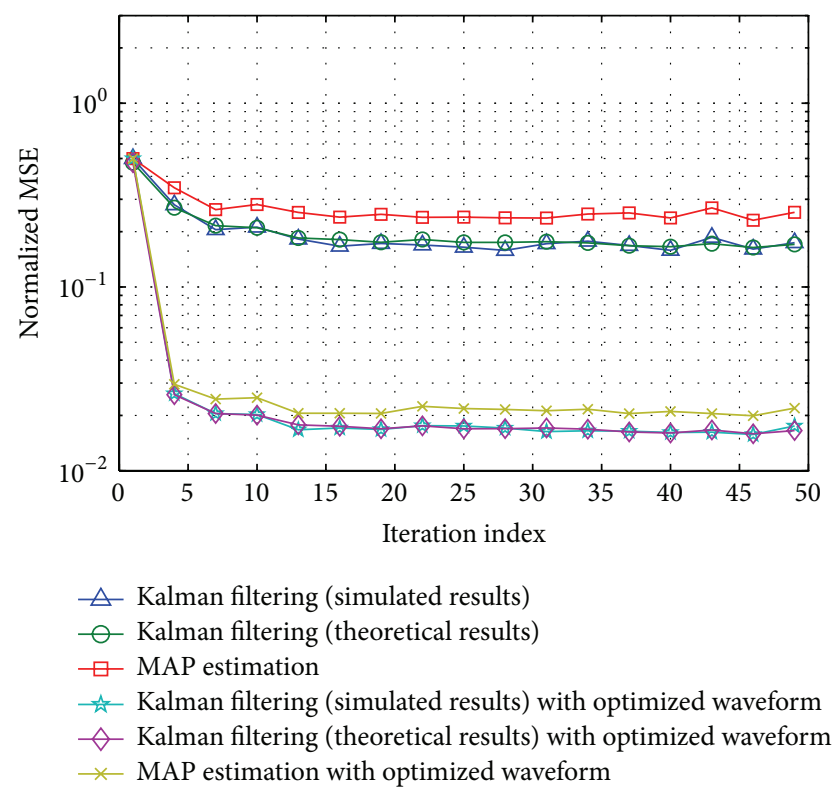

FIGURE 6: The comparison of estimation performance, including (a) the MAP approach with nonoptimized waveform; (b) the MAP approach with optimized waveform; (c) the KF approach with nonoptimized waveform; (d) the KF approach with optimized waveform.

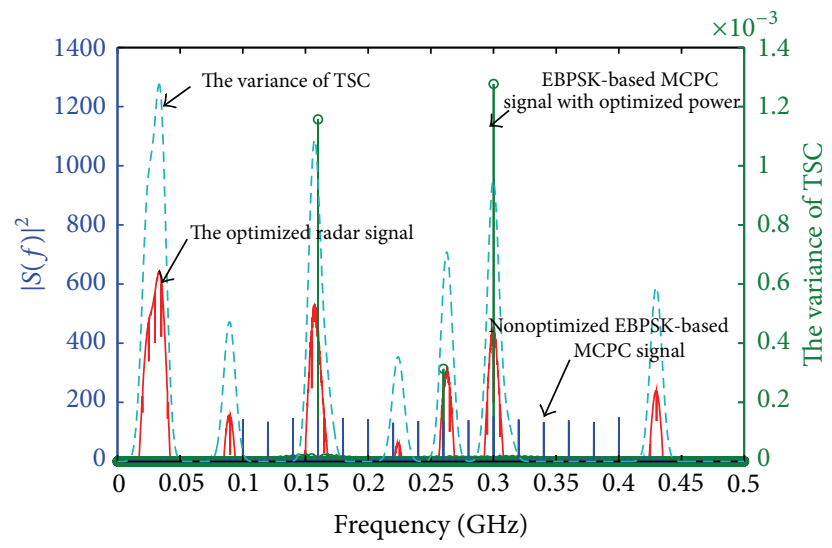

Figure 7: The spectrum of EBPSK-based MCPC signal with optimized power allocation.

with optimal spectrum, which can be calculated in (33). Figure 8 compares the normalized MSE of EBPSK-based MCPC signal with optimized power allocation with the one with uniform distributed power. We can observe that the estimation performance in the CRS can be improved significantly by optimizing the transmission power of each subcarrier, where the KF estimation with optimized power allocation of EBPSK-based MCPC signal achieves 7\% better than the one with uniform power allocation. Although the estimation performance of the optimized EBPSK-based MCPC signal is worse than the waveform with optimal spectrum (33), we can generate the optimal EBPSK-based MCPC signal by tuning the amplitudes of each subcarrier, which is more simple and convenient.

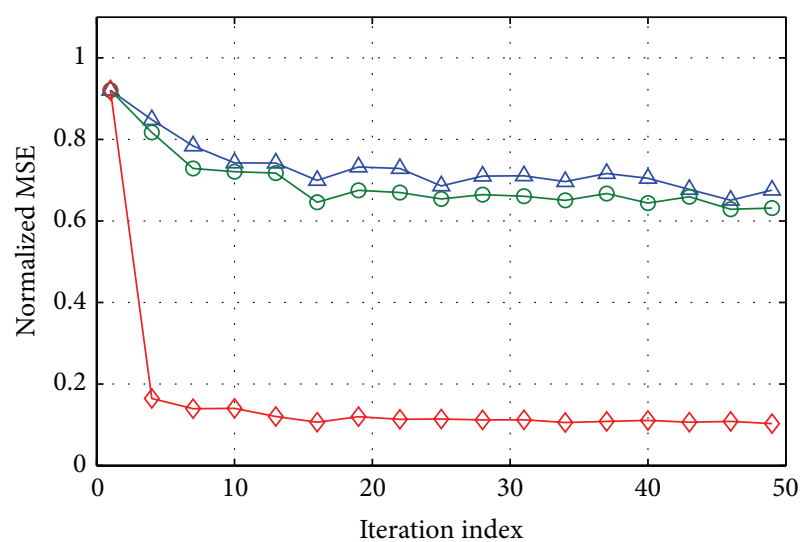

$\triangle$ Nonoptimized EBPSK-based MCPC signal
$\diamond-$ Optimal power of EBPSK-based MCPC signal
$\triangleleft$ Optimal radar signal

FIgURE 8: The comparison of estimation performance by $\mathrm{KF}$ approach, including (a) the EBPSK-based MCPC signal with uniform distributed power; (b) the EBPSK-based MCPC signal with optimized power allocation; (c) the optimal signal.

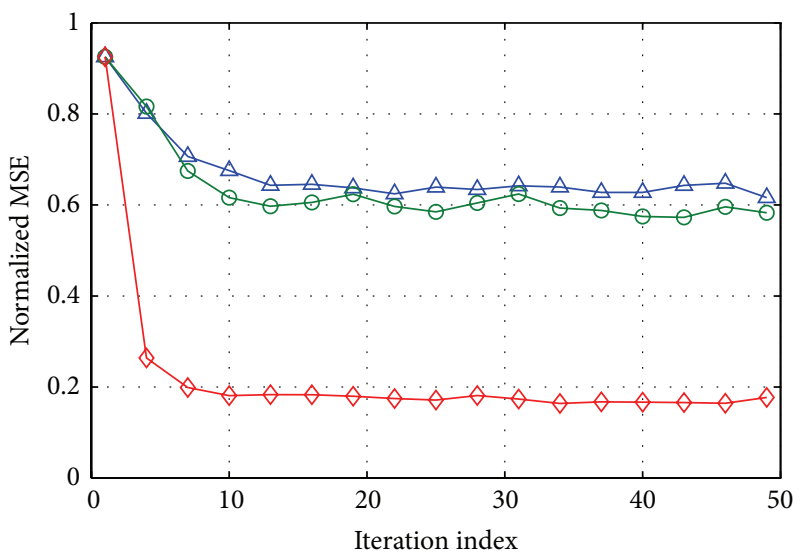

$\triangle$ Optimal power of EBPSK-based MCPC signal

$\odot$ Optimal power and coding matrix of EBPSK-based MCPC signal $\neg$ Optimal radar signal

FIGURE 9: Optimize the power and coding matrix of the EBPSKbased MCPC signal. The comparison of estimation performance by KF approach, including (a) the EBPSK-based MCPC signal with optimized power allocation and coding matrix; (b) the EBPSKbased MCPC signal with optimized power allocation; (c) the optimal signal.

Figure 9 compares the estimation performance of two types of optimized EBPSK-based MCPC signal, where the first one optimizes the power allocation and coding matrix simultaneously, and the second one only optimizes the power allocation. As the figure shows, the estimation performance is improved $8 \%$ by simultaneously optimizing these two parameters. Furthermore, the algorithm proposed in this work to optimize the coding matrix is easy to realize. 


\section{Conclusions}

In this work, we have investigated the problem of system optimization in the CRS with EBPSK-based MCPC signal, and a new radar working scheme has been proposed to take both the target detection and estimation into consideration. At the detection stage, we have deduced the theoretical threshold and the detection probability based on GLRT. At the estimation stage, the TSC is estimated by the approach based on KF. By exploiting the temporal correlation of TSC, the estimation performance of KF is $25 \%$ better than that of MAP. In addition, the transmitted waveform with optimal spectrum is obtained, which can improve the estimation performance of KF by $90 \%$. For the practical consideration, the iteration algorithms are proposed to optimize the EBPSKbased MCPC signal in terms of power allocation and coding matrix, which can achieve $7 \%$ and $8 \%$ performance improvement, respectively. In the future work, we will put our force on the system optimization of the temporal correlated CRS in the presence of clutter and jam.

\section{Conflict of Interests}

The authors declare that there is no conflict of interests regarding the publishing of this paper.

\section{Acknowledgment}

This work was supported in part by the National Natural Science Foundation of China (Grant no. 61271204) and the National Key Technology R\&D Program (Grant no. 2012BAH15B00).

\section{References}

[1] S. Haykin, "Cognitive radar: A way of the future," IEEE Signal Processing Magazine, vol. 23, no. 1, pp. 30-40, 2006.

[2] S. Haykin, Y. Xue, and T. N. Davidson, "Optimal waveform design for cognitive radar," in Proceedings of the 42nd Asilomar Conference on Signals, Systems and Computers (ASILOMAR '08), pp. 3-7, Pacific Grove, Calif, USA, October 2008.

[3] W. Huleihel, J. Tabrikian, and R. Shavit, "Optimal sequential waveform design for cognitive radar," in Proceedings of the IEEE International Conference on Acoustics, Speech, and Signal Processing, (ICASSP '12), pp. 2457-2460, Kyoto, Japan, March 2012.

[4] Y. Zhang, Z. Dong, L. Wu, and S. Wang, "A hybrid method for mri brain image classification," Expert Systems with Applications, vol. 38, no. 8, pp. 10049-10053, 2001.

[5] X. Zhang and C. Cui, "Range-spread target detecting for cognitive radar based on track-before-detect," International Journal of Electronics, vol. 101, no. 1, pp. 74-87, 2014.

[6] P. Setlur, N. Devroye, and Z. Cheng, "Waveform scheduling via directed information in cognitive radar," in Proceedings of the IEEE Statistical Signal Processing Workshop (SSP '12), pp. 864867, Ann Arbor, Mich, USA, August 2012.

[7] Y.-D. Zhang, L.-N. Wu, and G. Wei, "A new classifier for polarimetric SAR images," Progress in Electromagnetics Research, vol. 94, pp. 83-104, 2009.
[8] Y. Zhang and L. Wu, "Crop classification by forward neural network with adaptive chaotic particle swarm optimization," Sensors, vol. 11, no. 5, pp. 4721-4743, 2011.

[9] Y. Yu, A. P. Petropulu, and H. V. Poor, "Measurement matrix design for compressive sensing-based MIMO radar," IEEE Transactions on Signal Processing, vol. 59, no. 11, pp. 5338-5352, 2011.

[10] A. Leshem, O. Naparstek, and A. Nehorai, "Information theoretic adaptive radar waveform design for multiple extended targets," IEEE Journal on Selected Topics in Signal Processing, vol. 1, no. 1, pp. 42-55, 2007.

[11] S. P. Jacobs and J. A. O'Sullivan, "Automatic target recognition using sequences of high resolution radar range-profiles," IEEE Transactions on Aerospace and Electronic Systems, vol. 36, no. 2, pp. 364-381, 2000.

[12] M. R. Bell, "Information theory and radar waveform design," IEEE Transactions on Information Theory, vol. 39, no. 5, pp. 1578-1597, 1993.

[13] R. A. Romero, J. Bae, and N. A. Goodman, "Theory and application of SNR and mutual information matched illumination waveforms," IEEE Transactions on Aerospace and Electronic Systems, vol. 47, no. 2, pp. 912-927, 2011.

[14] F. Z. Dai, H. W. Liu, P. H. Wang, and S. Z. Xia, "Adaptive waveform design for range-spread target tracking," Electronics Letters, vol. 46, no. 11, pp. 793-794, 2010.

[15] B. Jiu, H. Liu, B. Chen, and Z. Liu, "Waveform design for wideband radar target recognition based on eigensubspace projection," IET Radar, Sonar and Navigation, vol. 7, no. 6, pp. 702-709, 2013.

[16] Y. Zhang, L. Wu, N. Neggaz, S. Wang, and G. Wei, "Remotesensing image classification based on an improved probabilistic neural network," Sensors, vol. 9, no. 9, pp. 7516-7539, 2009.

[17] X. Deng, C. Qiu, Z. Cao, M. Morelande, and B. Moran, "Waveform design for enhanced detection of extended target in signaldependent interference," IET Radar, Sonar and Navigation, vol. 6, no. 1, pp. 30-38, 2012.

[18] S. Kay, "Optimal signal design for detection of Gaussian point targets in stationary Gaussian clutter/reverberation," IEEE Journal on Selected Topics in Signal Processing, vol. 1, no. 1, pp. 31-41, 2007.

[19] Y. Zhang, B. Peterson, G. Ji, and Z. Dong, "Energy preserved sampling for compressed sensing MRI," Computational and Mathematical Methods in Medicine, vol. 2014, Article ID 546814, 12 pages, 2014.

[20] R. A. Romero and N. A. Goodman, "Waveform design in signaldependent interference and application to target recognition with multiple transmissions," IET Radar, Sonar and Navigation, vol. 3, no. 4, pp. 328-340, 2009.

[21] N. Levanon, "Multifrequency radar signals," in Proceedings of the IEEE International Radar Conference, pp. 683-688, Alexandria, Va, USA, May 2000.

[22] N. Levanon and E. Mozeson, "Multicarrier radar signal-Pulse train and CW," IEEE Transactions on Aerospace and Electronic Systems, vol. 38, no. 2, pp. 707-720, 2002.

[23] N. Levanon, "Multifrequency complementary phase-coded radar signal," IEE Proceedings: Radar, Sonar and Navigation, vol. 147, no. 6, pp. 276-284, 2000.

[24] S. Sen and C. W. Glover, "Optimal multicarrier phase-coded waveform design for detection of extended targets," in Proceedings of the IEEE Radar Conference, pp. 1-6, Ottawa, Canada, April-May 2013. 
[25] P. Chen and L. Wu, "Optimum weighted cumulation target detection for resonance region multi-carrier radar," in Proceedings of the International Conference on Wireless Communications and Signal Processing (WCSP '13), pp. 1-4, Hangzhou, China, October 2013.

[26] E. Mozeson and N. Levanon, "Multicarrier radar signals with low peak-to-mean envelope power ratio," IEE Proceedings: Radar, Sonar and Navigation, vol. 150, no. 2, pp. 71-77, 2003.

[27] $\mathrm{L}$. Wu, "The progress of the UNB speed communications," Progress in Natural Science, pp. 1467-1473, 2007.

[28] X. Chen and L. Wu, "A novel detection scheme for EBPSK system," Mathematical Problems in Engineering, vol. 2012, Article ID 956191, 14 pages, 2012.

[29] Y. Jin, L.-N. Wu, P.-K. Ying, and J. Yu, "RS coded EBPSKMODEM based on SVM discrimination," Journal of Applied Sciences, vol. 31, no. 1, pp. 48-52, 2013.

[30] P. Chen and L. Wu, "Compressive sensing for multi-carrier EBPSK radar," Journal of Southeast University (Natural Science Edition), vol. 44, no. 1, pp. 23-27, 2014.

[31] M. Feng, L. Wu, J. Ding, and C. Qi, "BER analysis and verification of EBPSK system in AWGN channe," IEICE Transactions on Communications, vol. 94, no. 3, pp. 806-809, 2011.

[32] D. Hammarwall, M. Bengtsson, and B. Ottersten, "Acquiring partial CSI for spatially selective transmission by instantaneous channel norm feedback," IEEE Transactions on Signal Processing, vol. 56, no. 3, pp. 1188-1204, 2008. 


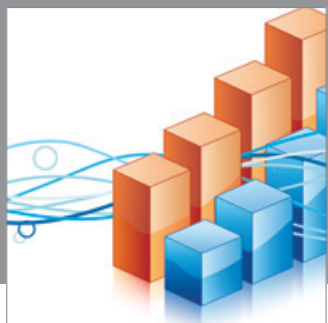

Advances in

Operations Research

mansans

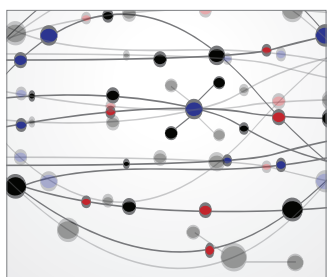

The Scientific World Journal
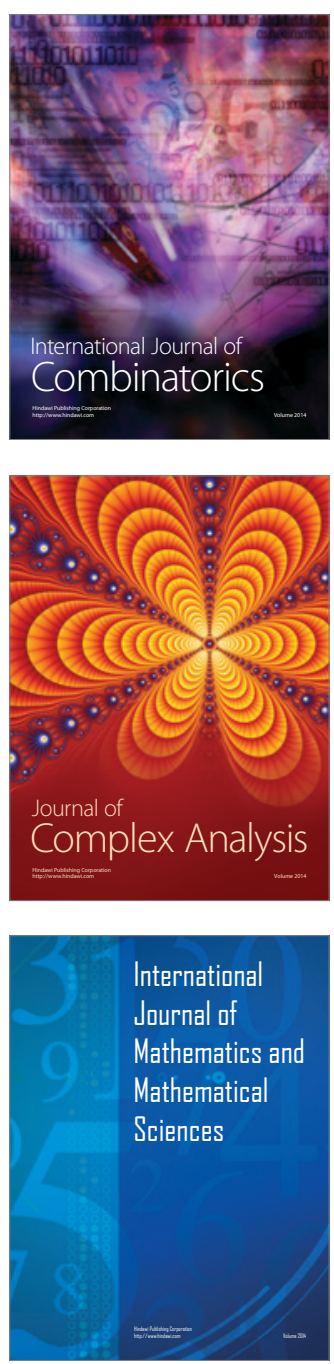
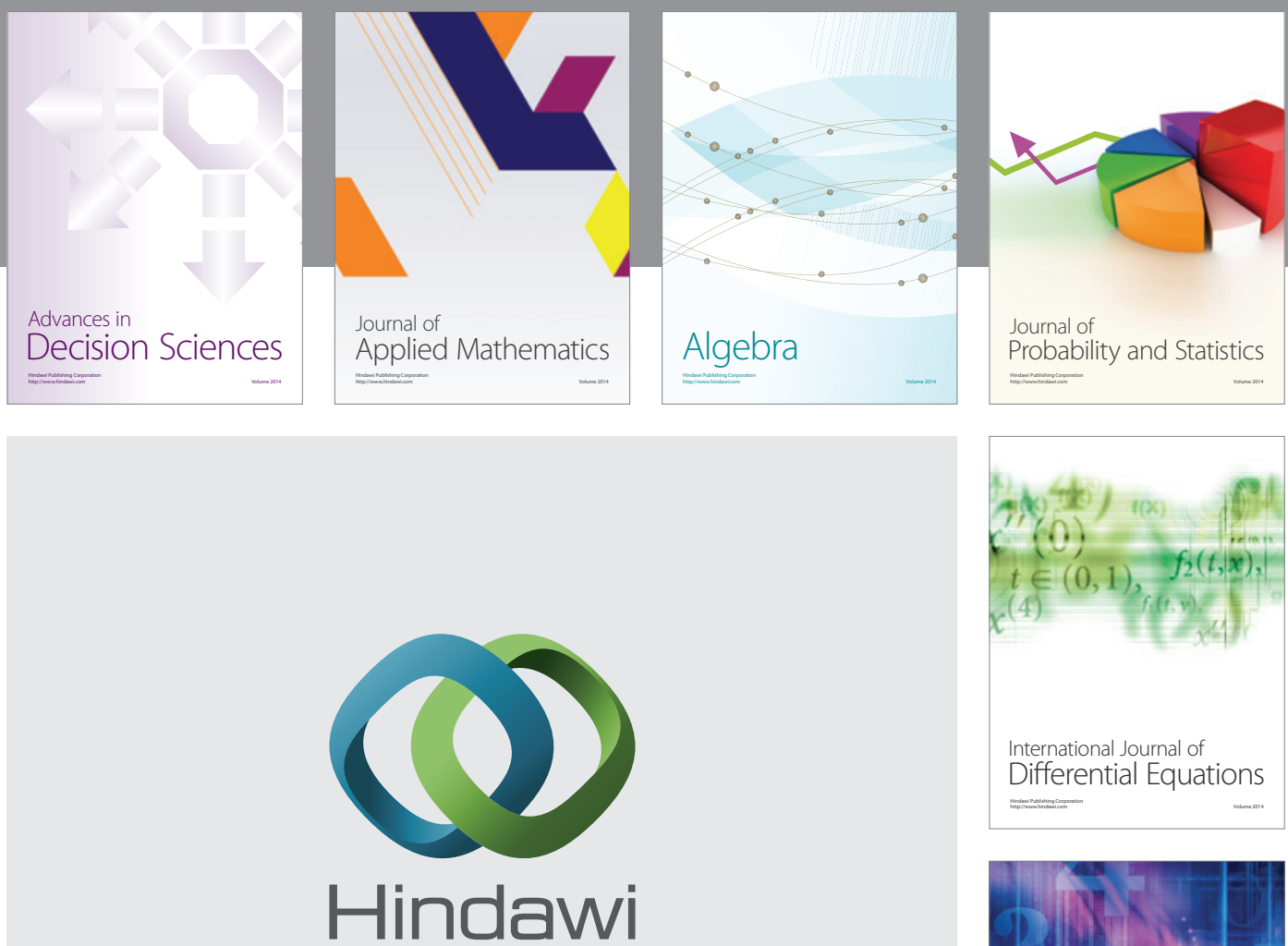

Submit your manuscripts at http://www.hindawi.com
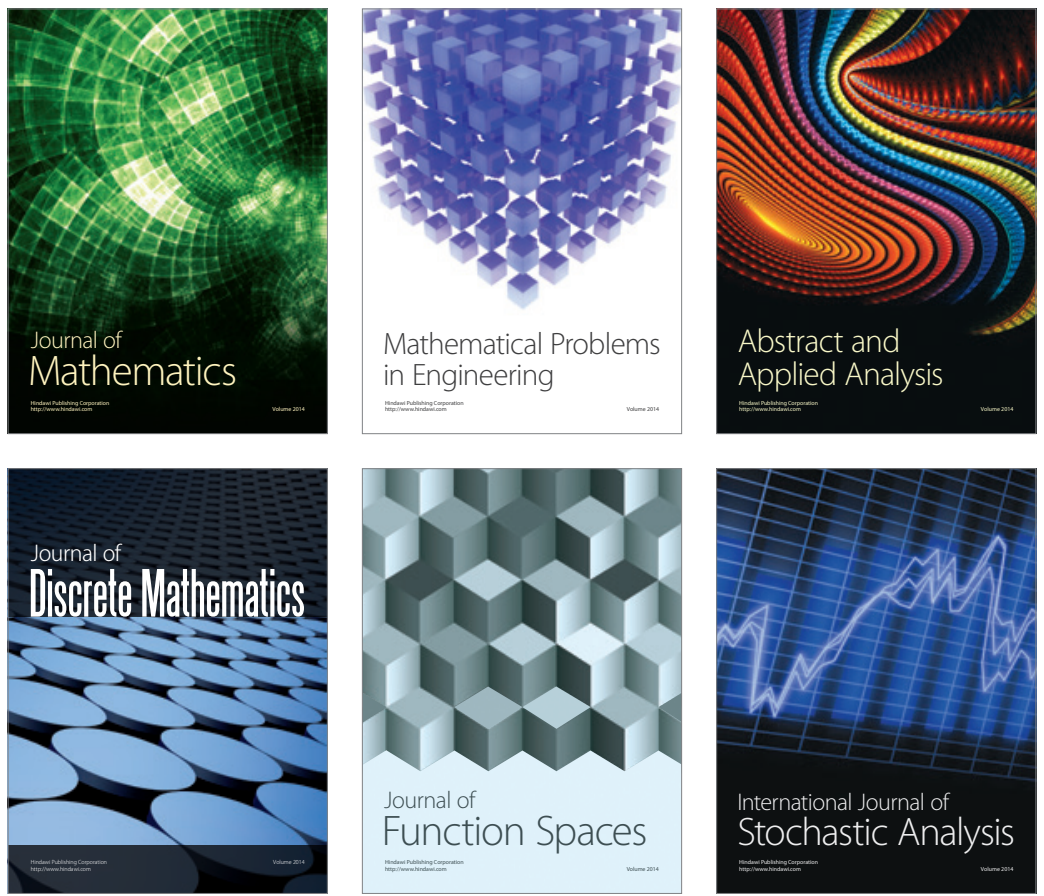

Journal of

Function Spaces

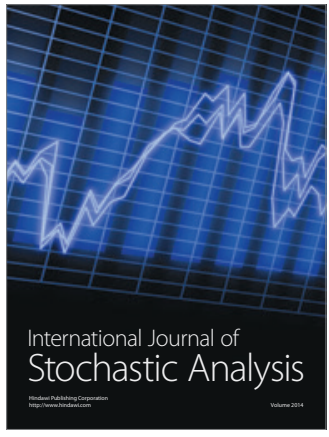

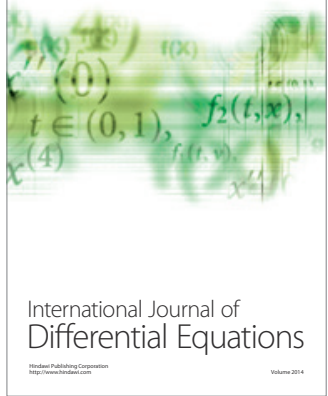
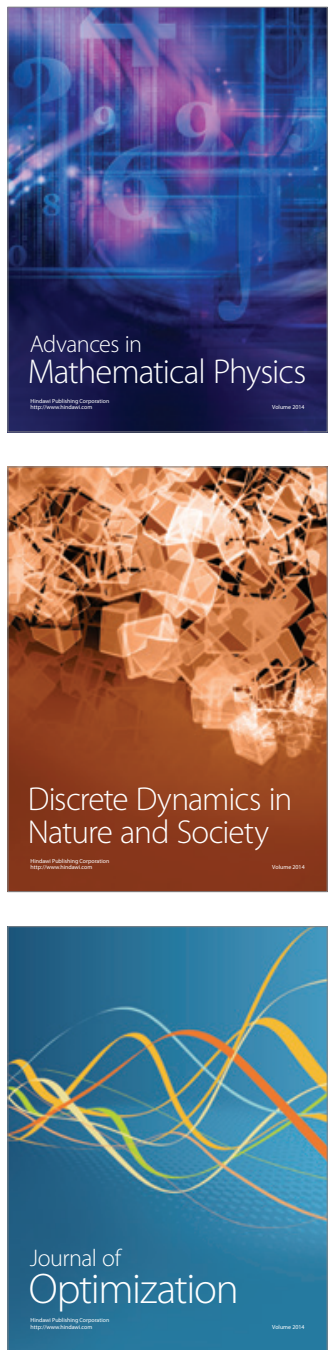